\title{
Simultaneous Graph Embeddings with Fixed Edges
}

\author{
Elisabeth Gassner ${ }^{2}$, Michael Jünger ${ }^{3}$, Merijam Percan ${ }^{3}$, Marcus Schaefer ${ }^{1}$, and \\ Michael Schulz ${ }^{3}$ \\ 1 DePaul University, School of CTI, 243 South Wabash, Ste 401, \\ 60604 Chicago, IL, USA, mschaefer@cs.depaul.edu \\ 2 Technische Universität Graz, Institut für Mathematik B, Steyrergasse 30/II, \\ 8010 Graz, Austria, gassner@opt.math.tu-graz.ac.at \\ 3 Universität zu Köln, Institut für Informatik, Pohligstraße 1, 50969 Köln, Germany, \\ \{mjuenger, percan, schulz\}@informatik. uni-koeln.de, partially supported by \\ Marie-Curie Research Training Network (ADONET) and by the German Science \\ Foundation (JU204/10-1).
}

\begin{abstract}
We study the problem of simultaneously embedding several graphs on the same vertex set in such a way that edges common to two or more graphs are represented by the same curve. This problem is known as simultaneously embedding graphs with fixed edges. We show that this problem is closely related to the weak realizability problem: Can a graph be drawn such that all edge crossings occur in a given set of edge pairs? By exploiting this relationship we can explain why the simultaneous embedding problem is challenging, both from a computational and a combinatorial point of view.

More precisely, we prove that simultaneously embedding graphs with fixed edges is NP-complete even for three planar graphs. For two planar graphs the complexity status is still open.
\end{abstract}

\section{Introduction}

Cologne offers various methods of public transport, including buses, trains, and even a cable car to the zoo. Suppose your task is to design a system map displaying all of the transport systems simultaneously.

We can easily model each transport system by itself as a graph: the bus system has bus stops as vertices and edges correspond to direct connections between two stops on a bus route. Similarly, for the train system we model train stations as vertices and connections between train stations as edges. Let us make the assumption that each system by itself (bus, train, cable car, etc.) is planar; for instance, two bus routes do not intersect except at bus stops. We also assume that all graphs share the same vertex set, so a bus stop can coincide with a train stop or a cable car stop. To draw the full system map we need to embed vertices and edges in the plane such that each of the systems - bus, train, cable car - is planar by itself, but allowing, for instance, train lines and bus routes to cross outside stations. 
More formally, a simultaneous embedding of a set of graphs sharing the same vertex set is a mapping of the vertices into the plane and a planar embedding of each graph on the vertex set [1]. Notice that edges belonging to different graphs are allowed to intersect. There are several natural ways to restrict the model; for example, we could require all edges to be drawn as straight-line segments. Such a drawing is called a simultaneous geometric embedding of the graphs [1].

Returning to the transport system example, it appears that asking for a simultaneous geometric embedding of the individual systems is too strict a requirement: we would be willing to accept Jordan curves to represent edges. However, requiring only a simultaneous embedding does not seem satisfactory either: if a train and bus route run in parallel, we would like to see them drawn in parallel; that is, the two routes should be represented by the same curve. This restriction leads to the notion of simultaneous embeddings with fixed edges introduced by Erten and Kobourov [2], and it is this notion that will mostly concern us in this paper: if several graphs share the same edge, that edge is represented by the same curve in the drawing. We see that every simultaneous geometric embedding is a simultaneous embedding with fixed edges, which, in turn, is a simultaneous embedding.

While different variants of simultaneous embeddings have been investigated from both theoretical and practical points of view [1-7], there is not much known about simultaneous embeddings with fixed edges. Erten and Kobourov [2] give an algorithm that constructs a simultaneous embedding with fixed edges of a tree and a path in linear time and uses at most one bend per edge. They point out that it is not known whether two trees can always be simultaneously embedded with fixed edges such that the number of bends is a small constant.

Studying the problem from a complexity-theoretic point of view gives a different challenge: it was not even clear whether the problem is decidable, let alone in NP or polynomial time. As we will see in Section 3 there is good reason for this: simultaneously embedding graphs with fixed edges is a different (and interesting) way to look at the weak realizability problem $[9,10]$. The complexity of the weak realizability problem is related to that of the string graph problem, whose complexity was only settled recently after being open for thirty years $[8$, 11]. The relationship implies that the simultaneous embedding problem can be decided in NP. We settle the computational complexity question for three or more graphs in Section 3 by showing that it is NP-complete. The complexity of the case for two graphs remains open.

\section{Preliminaries}

Given a graph $G=(V, E)$ and a set $R \subseteq\left(\begin{array}{c}E \\ 2\end{array}\right)=\{\{e, f\} \mid e, f \in E, e \neq f\}$, we call a drawing $D$ of $G$ in the plane in which all intersecting edge pairs belong to $R$ a weak realization of $(G, R)$.

Notice that not all edge pairs in $R$ are required to intersect in $D, R$ just defines the set of allowed crossings. $(G, R)$ is weakly realizable if such a drawing $D$ exists. 
Problem: $\quad$ Weak Realizability

Instance: $\quad$ A graph $G=(V, E)$ and a set $R \subseteq\left(\begin{array}{c}E \\ 2\end{array}\right)$.

Question: Is $(G, R)$ weakly realizable?

The weak realizability problem is closely related to the string graph problem [8]. Both problems are now known to be NP-complete even though this question was open for a long time [11].

The simultaneous embedding with fixed edges problem was introduced by Brass et al. [1]. It has become a major theoretical problem in simultaneous graph drawing. We will present an example of two planar graphs which do not have a simultaneous embedding with fixed edges. Brass et al already gave an example of two outerplanar graphs as well as three paths with no simultaneous embedding with fixed edges.

Problem: $\quad$ Simultaneous Embedding with Fixed Edges (SEFE)

Instance: $\quad$ A set of planar graphs $G_{i}=\left(V, E_{i}\right)$ on the same vertex set $V$.

Question: Are there plane drawings $D_{i}$ of $G_{i}$, such that each vertex is mapped to the same point in the plane in all $D_{i}$ and every shared edge $e \in E_{i} \cap E_{j}$ is represented by the same simple open Jordan curve in $D_{i}$ and $D_{j}$ ?

In Figure 1 two planar graphs $G_{1}, G_{2}$ are given for which no SEFE exist: the triangle induced by $v_{1}, v_{2}, v_{3}$ is equal to $G_{1} \cap G_{2}$ (visualized by bold edges) and makes it impossible to include the edge $\left(v_{4}, v_{5}\right) \in G_{1}$ into the unique embedding (up to a homomorphism of the plane) of $G_{2}$ without crossing an edge of $G_{1} \cap G_{2}$. This indicates that the SEFE problem is not trivial.
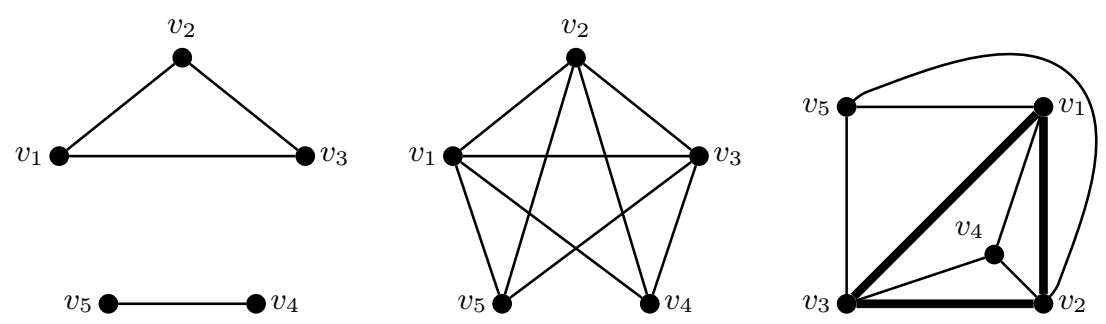

Fig. 1. Graph $G_{1}$ (left), graph $G_{2}$ (middle), unique planar embedding of $G_{2}$ (right).

\section{Complexity Results on Simultaneous Embeddings}

We start with a result dealing with the relationship between the two problems introduced in the previous section. This theorem implies the NP-completeness of SEFE in the general case. We will show later that the problem remains NPcomplete in the case of three graphs. 
Theorem 1. The weak realizability problem is polynomially equivalent to the problem of simultaneous embedding with fixed edges.

Proof. Let $((V, E), R)$ be an instance of the weak realizability problem. For every pair of edges $\{e, f\} \in\left(\begin{array}{c}E \\ 2\end{array}\right) \backslash R$ we construct a graph $G_{e, f}=(V,\{e, f\})$. It is easy to verify that the weak realizability problem is solvable if and only if there exists a simultaneous embedding with fixed edges for the set of constructed graphs $G_{e, f}$.

Let now $G_{i}=\left(V, E_{i}\right), i=1, \ldots, n$, be a set of graphs on the same vertex set $V$. Let $E=\bigcup_{i=1}^{n} E_{i}$ be the set of edges. Define $R$ as the set of those pairs of edges which are not contained in one graph $G_{i}$. Then the problem of simultaneously embedding the graphs $G_{i}$ is equivalent to the weak realizability problem of the pair $(G, R)$ with $G=(V, E)$.

Kratochvíl [8] and Schaefer, Sedgwick and Štefankovič [11] showed that weak realizability is NP-complete. Hence we get the following corollary.

Corollary 1. Simultaneous embedding with fixed edges is NP-complete.

In particular, SEFE lies in NP for every fixed number $k$ of graphs. As we will show next, it is even NP-complete in that case, as long as $k \geq 3$.

Theorem 2. Deciding whether three graphs have a simultaneous embedding with fixed edges is NP-complete.

Proof. We have already seen that SEFE for a fixed number of graphs is in NP. We will now show that there exists a polynomial transformation from 3SAT (which is well-known to be NP-complete) to SEFE for three planar graphs $G_{1}=\left(V, E_{1}\right)$, $G_{2}=\left(V, E_{2}\right)$ and $G_{3}=\left(V, E_{3}\right)$. Given an instance of 3SAT, we will construct an instance $\left(G_{1}, G_{2}, G_{3}\right)$ of SEFE. Then we will prove that the instance of 3SAT is satisfiable if and only if there exists a simultaneous embedding with fixed edges of $\left(G_{1}, G_{2}, G_{3}\right)$.

The decision problem 3SAT is given in the following way:

Problem: $\quad 3 S A T$

Instance: $\quad$ A set $U$ of boolean variables and a collection $C$ of clauses in conjunctive normal form over $U$ such that each clause $c \in C$ has exactly three literals.

Question: Is there a truth assignment to $U$ that satisfies $C$ ?

Construction: Let $C=\left\{c_{1}, \ldots, c_{m}\right\}$ be the set of clauses and let $U=$ $\left\{u_{1}, \ldots, u_{n}\right\}$ be the variable set of a 3SAT-instance. Each clause is of the form $c_{j}=l_{1}^{j} \vee l_{2}^{j} \vee l_{3}^{j}$ with literals either $l_{i}^{j}=u_{h}$ or $l_{i}^{j}=\bar{u}_{h}$ for some $h=1, \ldots, n$ and $i=1,2,3$.

Our construction of a SEFE-instance is made up of several components. For our construction we assume an ordering of the clauses, say $\left(c_{1}, c_{2}, \ldots, c_{m}\right)$. Furthermore we choose an order of the three literals in each clause $c_{j}$ and hence get an order of all literals in the following way $\left(l_{1}^{1}, l_{2}^{1}, l_{3}^{1}, l_{1}^{2}, \ldots, l_{3}^{m}\right)$. 
For each clause $c_{j}$ we define a clause box by introducing vertices $r_{1}^{j}, \ldots, r_{6}^{j}$, $y^{1, j}, y^{2, j}, y^{3, j}$. These vertices are connected by edges in $E_{2}$ and $E_{3}$ to form the cycle $\left(r_{1}^{j}, \ldots, r_{6}^{j}, y^{3, j}, y^{2, j}, y^{1, j}\right)$.

Next, we introduce two global vertices $R_{1}$ and $R_{2}$. We add an edge $\left(R_{1}, R_{2}\right)$ which is part of all three graphs $G_{1}, G_{2}$ and $G_{3}$. Furthermore, $R_{1}$ is connected to each clause box by four edges to the vertices $r_{2}^{j}, r_{3}^{j}, r_{4}^{j}$ and $r_{5}^{j}$. These edges are part of all three graphs. $R_{2}$ is connected to each clause gadget by two edges to vertices $r_{1}^{j}$ and $r_{6}^{j}$ in $E_{3}$.

To make the graphs more rigid we glue together neighboring clause boxes. This is done by identifying $r_{2}^{j+1}$ with $r_{5}^{j}$ and $r_{1}^{j+1}$ with $r_{6}^{j}$ for $j=1, \ldots, m-1$. Figure 2 shows the construction so far. We remark that all edges that have been constructed so far belong (among others) to $E_{3}$.

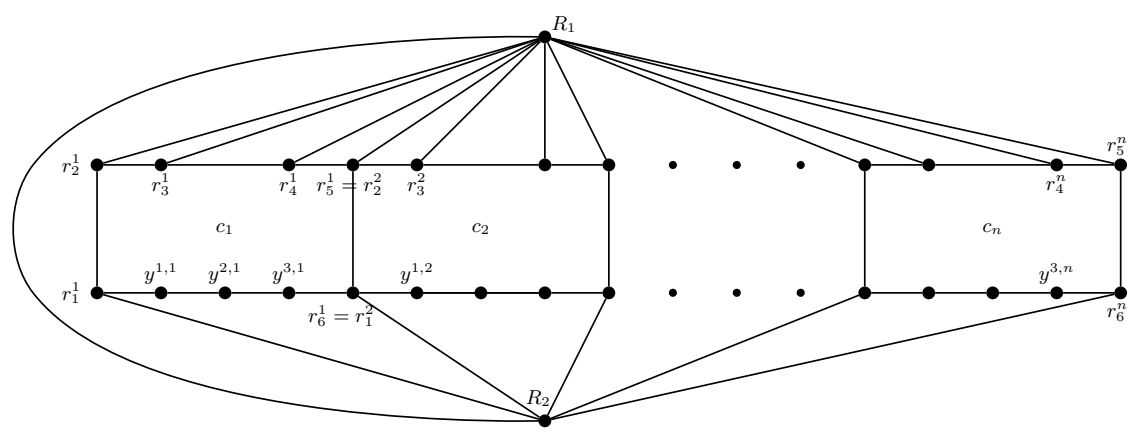

Fig. 2. The figure shows all clause boxes, the global vertices $R_{1}$ and $R_{2}$ and all connecting edges of $E_{3}$.

For every literal $l_{i}^{j}$ in clause $c_{j}$ we define a literal gadget consisting of the vertices $V^{i, j}=\left\{x_{k}^{i, j}, z_{k}^{i, j} \mid k=1, \ldots, 6\right\} \cup\left\{y^{i, j}\right\}$. The edge set of the gadget is shown in Figure 3. From now on edges of $G_{1}$ are illustrated by solid lines, edges of $G_{2}$ are dashed and edges of $G_{3}$ are dotted.

Furthermore, let $l_{i(1)}^{j(1)}, \ldots, l_{i\left(\omega_{h}\right)}^{j\left(\omega_{h}\right)}$ be all literals that belong to a variable $u_{h}$, that is $l_{i(\alpha)}^{j(\alpha)}=u_{h}$ or $l_{i(\alpha)}^{j(\alpha)}=\bar{u}_{h}$ for $\alpha=1, \ldots, \omega_{h}$. Assume that the literals are given in the order defined above. We connect each pair of adjacent literals in this ordered list by the following edges of $E_{2}$ (see Figure 4):

$$
\left(z_{1}^{i(k), j(k)}, z_{6}^{i(k+1), j(k+1)}\right),\left(z_{2}^{i(k), j(k)}, z_{5}^{i(k+1), j(k+1)}\right),\left(z_{3}^{i(k), j(k)}, z_{4}^{i(k+1), j(k+1)}\right)
$$

with $k=1, \ldots, \omega_{h}-1$.

For each clause we define a clause gadget consisting of three literal gadgets, the clause box and some additional vertices and edges. Let $c_{j}$ be a clause with literals $l_{1}^{j}, l_{2}^{j}$ and $l_{3}^{j}$. Notice that the three literal gadgets are already connected to the clause box via the vertices $y^{i, j}$ with $i=1,2,3$. Further connections are given by the additional edges $\left(r_{3}^{j}, x_{2}^{1, j}\right) \in E_{3}$ and $\left(r_{4}^{j}, x_{2}^{3, j}\right) \in E_{2}$. We also add two vertices $s^{j}, t^{j}$. 


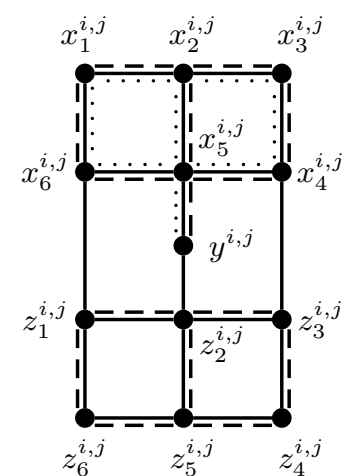

(a) Case $u_{h}=$ true

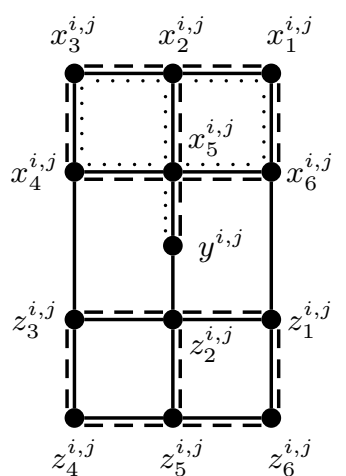

(b) Case $u_{h}=$ false

Fig. 3. Literal gadget for literal $l_{i}^{j}$ (with corresponding variable $u_{h}$ ) of clause $c_{j}$. The edges in $E_{1}$ are solid, those in $E_{2}$ are dashed and those in $E_{3}$ are dotted. The two different drawings (a) and (b) will become important later.

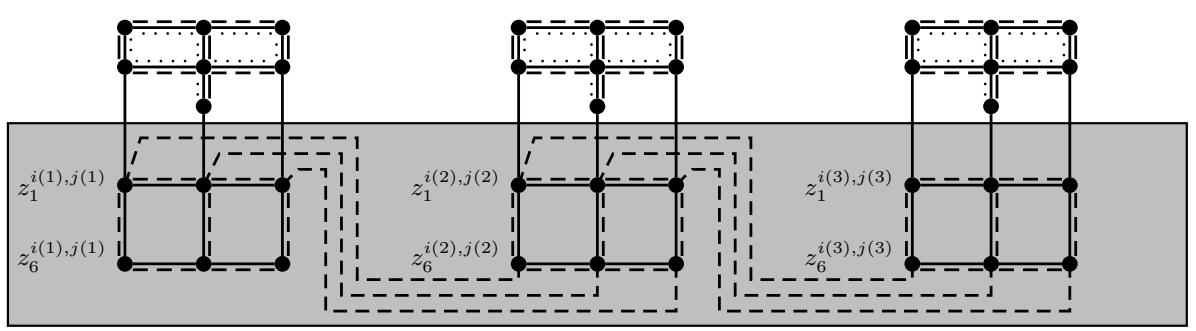

Fig. 4. Three literal gadgets that belong to variable $u_{h}$ are linked with edges in $E_{2}$.

In order to distinguish between negated and non-negated variables we define connections between the literal gadgets. The gadget of $l_{1}^{j}$ is connected along $s^{j}$ with the gadget of $l_{2}^{j}$. And the gadget of $l_{2}^{j}$ is connected along $t^{j}$ with the gadget of $l_{3}^{j}$. More precisely, we add edges $\left(p_{1}^{j}, s^{j}\right) \in E_{3}$ (where $p_{1}^{j}$ is a vertex of the gadget of $\left.l_{1}^{j}\right),\left(s^{j}, p_{2}^{j}\right) \in E_{1} \cap E_{2}$ and $\left(q_{2}^{j}, t^{j}\right) \in E_{1} \cap E_{3}$ (where $p_{2}^{j}$ and $q_{2}^{j}$ are vertices of the gadget of $u_{i_{2}}$ ) and $\left(t^{j}, q_{3}^{j}\right) \in E_{2}$ (where $q_{3}^{j}$ is a vertex of the gadget of $l_{3}^{j}$ ). If $l_{1}^{j}$ is not negated, we set $p_{1}^{j}=x_{3}^{1, j}$ (i. e., $x_{3}^{1, j}$ is connected with $s^{j}$ ), otherwise, if $l_{1}^{j}$ is negated, we set $p_{1}^{j}=x_{1}^{1, j}$. Analogously we set $p_{2}^{j}=x_{1}^{2, j}$ and $q_{2}^{j}=x_{3}^{2, j}$ if $l_{2}^{j}$ is not negated and $p_{2}^{j}=x_{3}^{2, j}$ and $q_{2}^{j}=x_{1}^{2, j}$ if $l_{2}^{j}$ is negated. And finally, we set $q_{3}^{j}=x_{1}^{3, j}$ if $l_{3}^{j}$ is not negated and $q_{3}^{j}=x_{3}^{3, j}$ if $l_{3}^{j}$ is negated. See Figure 5 for a clause gadget.

We complete the construction by adding an edge from $R_{2}$ to $z_{5}^{i, j}$ to graph $G_{3}$ for each literal $l_{i}^{j}$.

1. Assume that the 3 SAT-instance is satisfiable. We will prove that there exists a simultaneous embedding with fixed edges of the constructed instance. 


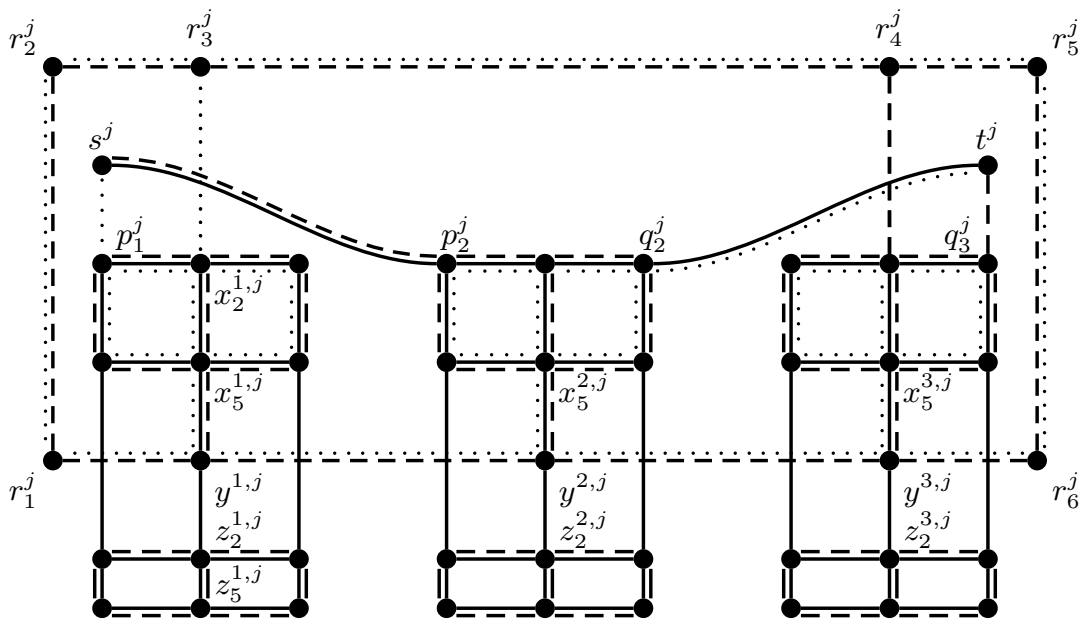

Fig. 5. Clause gadget for the case where $u_{k(2)}$ is the only variable that makes $c_{j}$ true.

If variable $u_{h}=$ true we draw each of its literal gadgets as shown in Figure 3(a). Otherwise we draw the gadgets as shown in Figure 3(b).

The clause gadgets are drawn side by side as illustrated in Figure 2 for the clause boxes. Furthermore, the $x$-vertices of each literal gadget lie inside the cycle of the corresponding clause boxes and the $z$-vertices lie outside (see Figure 5). Moreover, every variable gets its own horizontal level for the $z$-variables of its literal gadgets (in Figure 4 the horizontal level is marked gray). Since all literal gadgets that belong to one variable $u_{h}$ are drawn in the same way we can draw the edges that connect literal gadgets which belong to the same variable without crossings (see Figure 4).

It remains to show that we can draw the edges inside the clause gadgets without crossings of edges of the same graph. We say that a variable $u$ makes a clause $c$ true if either $u \in c$ and $u=\operatorname{true}$ or if $\bar{u} \in c$ and $u=\mathrm{false}$. Since the instance of 3SAT is satisfiable there exists at least one variable $u$ in each clause $c$ that makes $c$ true.

Consider clause $c_{j}$ with literals $l_{1}^{j}, l_{2}^{j}$, and $l_{3}^{j}$. Let $u_{k(i)}$ be the corresponding variable to literal $l_{i}^{j}$, thus either $l_{i}^{j}=u_{k(i)}$ or $l_{i}^{j}=\bar{u}_{k(i)}$. Assume first that $u_{k(1)}$ makes $c_{j}$ true. Then there are two possibilities. Either $u_{k(1)} \in c_{j}$ and $u_{k(1)}$ is set to true or $\bar{u}_{k(1)} \in c_{j}$ and $u_{k(1)}=$ false. In the first case the literal gadget is drawn like Figure $3(\mathrm{a})$ and $p_{1}^{j}=x_{3}^{1, j}$ and in the other case we have the situation of Figure $3(\mathrm{~b})$ and $p_{1}^{j}=x_{1}^{1, j}$. But in both of these cases the vertex $p_{1}^{j}$ is the upper right vertex of literal gadget $l_{1}^{j}$ (see Figure 6). Figure 6 shows that in the case where $u_{k(1)}$ is the only variable that makes $c_{j}$ true there is a simultaneous embedding with fixed edges of the corresponding clause gadget. Simple modifications yield a simultaneous embedding for the case where $u_{k(1)}$ is not the only variable that makes $c_{j}$ true. Due to symmetry an analogue drawing can be found for the case where $u_{k(3)}$ makes $c_{j}$ true. 


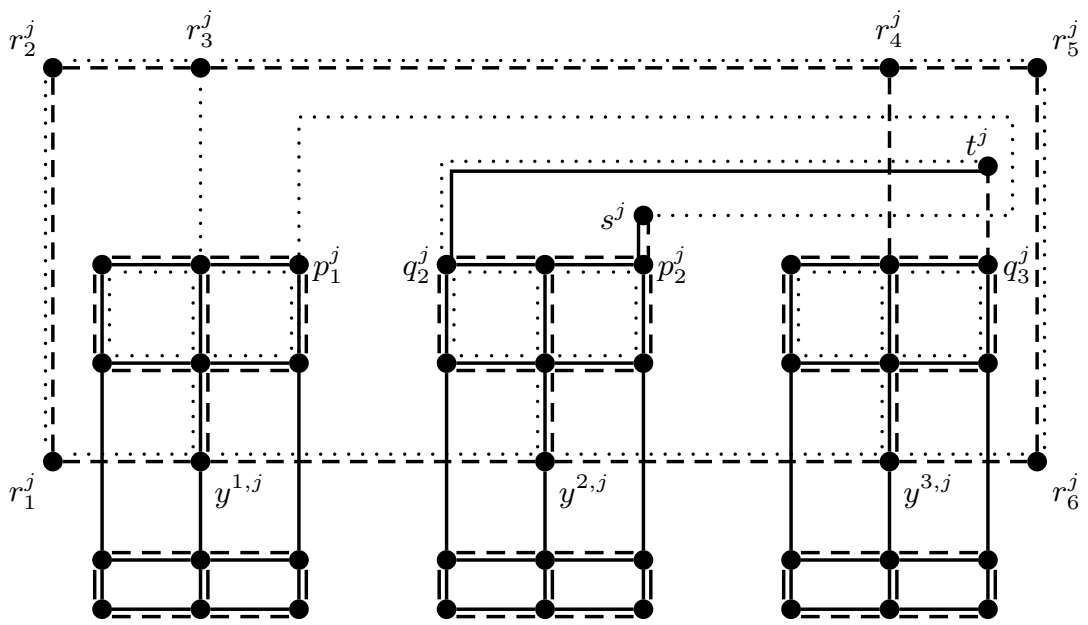

Fig. 6. Simultaneous embedding of the clause gadget when $u_{k(1)}$ is the only variable that makes $c_{j}$ true.

Finally, if $u_{k(2)}$ makes $c_{j}$ true we can find a simultaneous embedding as shown in Figure 5. Hence, we have found a simultaneous embedding with fixed edges of the constructed instance.

2. Now assume that we are given a simultaneous embedding with fixed edges. We will show that we can find a satisfying truth assignment for the 3SATinstance.

Notice that the subgraph of $G_{3}$ shown in Figure 2 is a triconnected subdivision. Consequently, it has a unique combinatorial embedding. We choose the planar embedding with the edge $\left(R_{1}, R_{2}\right)$ on the boundary of the outer face such that the cycle $\left(R_{1}, r_{2}^{1}, r_{1}^{1}, R_{2}, r_{6}^{n}, r_{5}^{n}\right)$ has the same order as visualized in Figure 2.

Due to the edge $\left(R_{2}, z_{5}^{i, j}\right)$ the vertex $z_{5}^{i, j}$ lies outside the clause box of clause $c_{j}$ for each literal $l_{i}^{j}$. Hence, all $z$-vertices of every literal lie outside all clause boxes.

Moreover, all $x$-vertices of a literal $l_{i}^{j}$ lie inside the clause box of clause $c_{j}$. Notice first that both paths $\left(r_{3}^{j}, x_{2}^{1, j}, x_{5}^{1, j}, y^{1, j}\right)$ and $\left(r_{4}^{j}, x_{2}^{3, j}, x_{5}^{3, j}, y^{3, j}\right)$ have to be completely within the clause box of clause $c_{j}$ due to vertex $R_{1}$ and its incident edges. Then all $x_{k}^{1, j}$ and $x_{k}^{3, j}, k=1, \ldots, 6$, have to lie within the clause box. Thus, $s^{j}$ is also forced to lie within the clause box. Otherwise, the edge $\left(p_{1}^{j}, s^{j}\right) \in E_{3}$ crosses an edge of the clause box boundary which is also in $E_{3}$. Hence, the edge $\left(s^{j}, p_{2}^{j}\right)$ and then all $x_{k}^{2, j}, k=1, \ldots, 6$, have to lie within the clause box, otherwise there would be a crossing of two edges of graph $G_{2}$.

Observe further, that each literal gadget contains (among others) two cycles, $C_{1}^{i, j}=\left\{x_{k}^{i, j} \mid k=1, \ldots, 6\right\}$ and $C_{2}^{i, j}=\left\{z_{k}^{i, j} \mid k=1, \ldots, 6\right\}$. Since the literal gadgets in $G_{1}$ are planar graphs, that are a triconnected subdivision, these gadgets have a unique planar embedding. Therefore, the clockwise ordering of the vertices of $C_{1}^{i, j}$ and $C_{2}^{i, j}$ in a simultaneous embedding is either $\left(x_{1}^{i, j}, x_{2}^{i, j}, \ldots, x_{6}^{i, j}\right)$ and 
$\left(z_{1}^{i, j}, z_{2}^{i, j}, \ldots, z_{6}^{i, j}\right)$ or $\left(x_{6}^{i, j}, x_{5}^{i, j}, \ldots, x_{1}^{i, j}\right)$ and $\left(z_{6}^{i, j}, z_{5}^{i, j}, \ldots z_{1}^{i, j}\right)$. In the first case, we say the literal gadget has a positive ordering and in the second case it has a negative ordering. Thus, the clockwise ordering of $C_{1}^{i, j}$ implies the clockwise ordering of $C_{2}^{i, j}$ and vice versa.

Again, let $l_{i(1)}^{j(1)}, \ldots, l_{i\left(\omega_{h}\right)}^{j\left(\omega_{h}\right)}$ be all literals that belong to the same variable $u_{h}$. Notice that the subgraph of $G_{2}$ induced by the vertices $z_{q}^{i(k), j(k)}$ for $q=1, \ldots, 6$ and $k=1, \ldots, \omega_{h}$ is also a triconnected subdivision. Since $G_{2}$ is drawn planar, the ordering of all cycles $C_{2}^{i(k), j(k)}$ has to be the same. We conclude that all cycles $C_{1}$ and $C_{2}$ that belong to one variable $u_{h}$ have the same ordering. Therefore, we can let

$$
u_{h}= \begin{cases}\text { true } & \text { if the literal gadgets of } u_{h} \text { have a positive ordering } \\ \text { false } & \text { if the literal gadgets of } u_{h} \text { have a negative ordering. }\end{cases}
$$

We define the interior of cycle $C^{\prime}=\left(r_{1}^{j}, r_{2}^{j}, r_{3}^{j}, x_{2}^{1, j}, x_{5}^{1, j}, y^{1, j}\right)$ as region $F_{1}^{j}$, the interior of cycle $\left(r_{3}^{j}, r_{4}^{j}, x_{2}^{3, j}, x_{5}^{3, j}, y^{3, j}, y^{2, j}, y^{1, j}, x_{5}^{1, j}, x_{2}^{1, j}\right)$ as region $F_{2}^{j}$ and the interior of cycle $C^{\prime \prime}=\left(r_{4}^{j}, r_{5}^{j}, r_{6}^{j}, y^{3, j}, x_{5}^{3, j}, x_{2}^{3, j}\right)$ as region $F_{3}^{j}$ (see Figure 7 ).

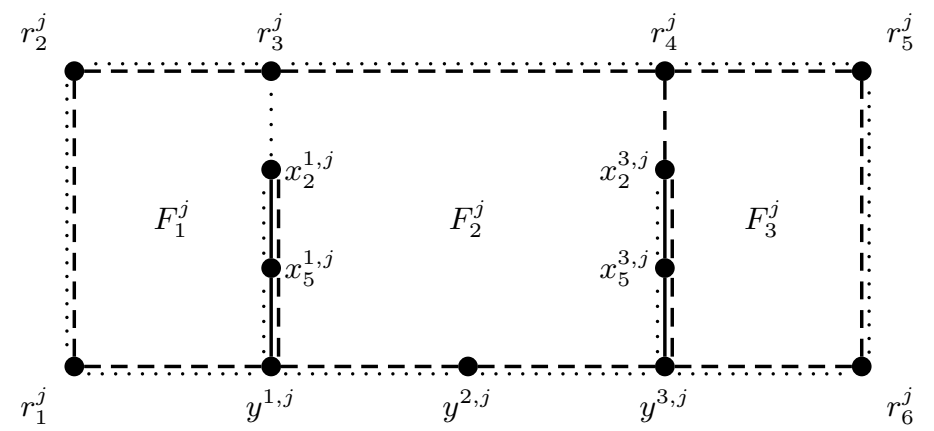

Fig. 7. $p_{1}^{j}$ and $s^{j}$ have to be embedded in region $F_{1}^{j}, p_{2}^{j}$ and $q_{2}^{j}$ have to be embedded in region $F_{2}^{j}$ and $q_{3}^{j}$ and $t^{j}$ have to be embedded in region $F_{3}^{j}$.

Assume that there exists a clause $c_{j}$ that is not true. This implies that none of the three corresponding clause variables $u_{k(1)}, u_{k(2)}, u_{k(3)}$ makes $c_{j}$ true. If $u_{k(1)} \in c_{j}$ then the literal gadget of $l_{1}^{j}$ has a negative ordering and $p_{1}^{j}=x_{3}^{1, j}$ is connected with $s^{j}$ (see Figure 8). Otherwise, if $\bar{u}_{k(1)} \in c_{j}$ then the variable gadgets of $u_{k(1)}$ have a positive ordering and $p_{1}^{j}=x_{1}^{1, j}$ is connected with $s^{j}$. But in both cases the vertex $p_{1}^{j}$ has to be embedded in region $F_{1}^{j}$. With similar arguments for $u_{k(2)}$ and $u_{k(3)}$ we conclude that the clause gadget of $c_{j}$ is of the form illustrated in Figure 8. To be more specific, vertex $q_{3}^{j}$ has to lie within region $F_{3}^{j}$ and both $p_{2}^{j}$ and $q_{2}^{j}$ have to be embedded within region $F_{2}^{j}$. For the last two vertices we have an additional restriction: running through cycle $C_{1}^{2, j}$ 
in clockwise order starting from vertex $x_{5}^{2, j}$ the vertex $p_{2}^{j}$ comes first compared to vertex $q_{2}^{j}$ (see Figure 8).

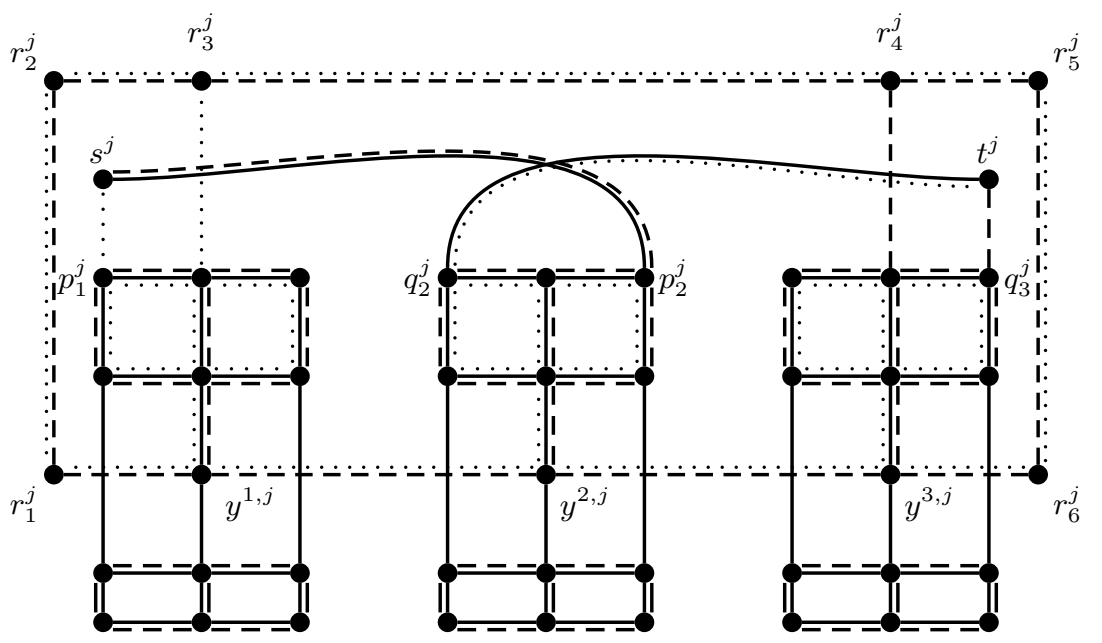

Fig. 8. If a clause is false then there exists a crossing of two edges of the same graph in the corresponding clause gadget.

In Figure 8 we observe that vertex $s^{j}$ has to be placed within region $F_{1}^{j}$. Otherwise edge $\left(p_{1}^{j}, s^{j}\right) \in E_{3}$ would cross at least one edge of the cycle $C^{\prime} \subset E_{3}$. Moreover, $t^{j}$ has to be placed within the cycle $C^{\prime \prime}$ that is region $F_{3}^{j}$. Otherwise we have a crossing of two edges of $E_{2}$, since $C^{\prime \prime} \subseteq E_{2}$. If $\left(q_{2}^{j}, t^{j}\right)$ is drawn without crossing an edge of the same graph there exists a cycle consisting of edges in $E_{1} \cup E_{2}$ that separates $p_{2}^{j}$ from $s^{j}$. Since $\left(s^{j}, p_{2}^{j}\right) \in E_{1} \cap E_{2}$ we conclude that there exists a crossing of at least two edges of the same graph. If $\left(s^{j}, p_{2}^{j}\right)$ is drawn, analogue implications lead to a non planar embedding of one of the graphs $G_{1}$, $G_{2}$ or $G_{3}$. This leads to a contradiction to the assumption that clause $c_{j}$ is not true. Hence, all clauses are satisfied and thus, we have found a truth assignment.

\section{Summary and Future Work}

We believe that in spite of their unwieldy name, simultaneous graph embeddings with fixed edges are a natural problem to study. Erten and Kobourov [2] supply an important reason why the fixed-edge model is more natural for many applications in comparison to the unrestricted version: Building a mental model of a complex graph drawing is easier if the underlying structure resembles the graph drawing, or, as Charles Peirce would have phrased it, if the drawing is iconic. In other words, edges belonging to several graphs should not have multiple representations, since this complicates forming a mental model: we have to identify 
the different curves as representations of the same edge. And while straightline embeddings resolve this issue, they exclude the possibility of simultaneously embedding even very simple planar graphs, such as trees [5].

It has been known that the weak realizability problem is related to several famous problems, such as string graphs, topological inference, and Euler diagrams $[9,11]$, but the connection to fixed edge embeddings is the most purely combinatorial connection we have seen so far.

The main open question of complexity is the question whether two planar graphs allow a simultaneous embedding with fixed edges.

\section{Acknowledgments}

We would like to thank Dániel Marx and Stephen G. Kobourov for helpful discussions. Moreover, we are grateful to Stefan Hachul and Alejandro Estrella Balderrama for proof-reading this paper.

\section{References}

1. Peter Brass, Eowyn Cenek, Christian A. Duncan, Alon Efrat, Cesim Erten, Dan Ismailescu, Stephen G. Kobourov, Anna Lubiw, and Joseph S. B. Mitchell. On simultaneous planar graph embeddings. In Workshop on Algorithms and Data Structures. Lecture Notes in Computer Science, volume 2748, pages 243-255. SpringerVerlag, 2003.

2. Cesim Erten and Stephen G. Kobourov. Simultaneous embedding of planar graphs with few bends. In 12th Intl. Symp. on Graph Drawing. Lecture Notes in Computer Science, volume 3383, pages 195-205. Springer-Verlag, 2004.

3. Cesim Erten and Stephen G. Kobourov. Simultaneous embedding of a planar graph and its dual on the grid. Theory of Computing Systems, 38:313-327, 2005.

4. Cesim Erten, Stephen G. Kobourov, Vu Le, and Armand Navabi. Simultaneous graph drawing: Layout algorithms and visualization schemes. In 11th Intl. Symp. on Graph Drawing. Lecture Notes in Computer Science, volume 2912, pages 437449. Springer-Verlag, 2003.

5. Markus Geyer, Michael Kaufmann, and Imrich Vrt'o. Two trees which are selfintersecting when drawn simultaneously. In 13th Intl. Symp. on Graph Drawing. Lecture Notes in Computer Science, volume 3843, pages 201-210. Springer-Verlag, 2005.

6. Emilio Di Giacomo and Giuseppe Liotta. A note on simultaneous embedding of planar graphs. In 21st European Workshop on Comp.Geometry, pages 207-210, 2005.

7. Stephen G. Kobourov and Chandan Pitta. An interactive multi-user system for simultaneous graph drawing. In 12th Intl. Symp. on Graph Drawing. Lecture Notes in Computer Science, volume 3383, pages 492-501. Springer-Verlag, 2004.

8. Jan Kratochvíl. String graphs II Recognizing string graphs is NP-hard. Journal of Combinatorial Theory, B 52:67-78, 1991.

9. Jan Kratochvíl. Crossing number of abstract topological graphs. In 6th Intl. Symp. on Graph Drawing. Lecture Notes in Computer Science, volume 1547, pages 238245. Springer-Verlag, 1998. 
10. Jan Kratochvíl, Anna Lubiw, and Jaroslav Nešetřil. Noncrossing subgraphs in topological layouts. SIAM Journal on Discrete Mathematics, 4(2):223-244, May 1991.

11. Marcus Schaefer, Eric Sedgwick, and Daniel Štefankovič. Recognizing string graphs in NP. Journal Comput. Syst. Sci., 67(2):365-380, 2003. 